\title{
Thermal effects on the stability of excited atoms in cavities
}

\author{
F. C. Khanna* \\ Department of Physics, University of Alberta, Edmonton, Alberta T6G 2J1, Canada, \\ and TRIUMF, Vancouver, British Columbia V6T 2A3, Canada
}

A. P. C. Malbouisson ${ }^{\dagger}$

Centro Brasileiro de Pesquisas Físicas/MCT, 22290-180 Rio de Janeiro, Rio de Janeiro, Brazil

J. M. C. Malbouisson

Instituto de Física, Universidade Federal da Bahia, 40.210-310 Salvador, Bahia, Brazil

\author{
A. E. Santana $a^{\S}$ \\ Instituto de Física, Universidade de Brasília, 70910-900 Brasília, Distrito Federal, Brazil, \\ and Department of Physics, University of Alberta, Edmonton, Alberta T6G 2J1, Canada
}

(Received 8 September 2009; published 22 March 2010)

\begin{abstract}
An atom, coupled linearly to an environment, is considered in a harmonic approximation in thermal equilibrium inside a cavity. The environment is modeled by an infinite set of harmonic oscillators. We employ the notion of dressed states to investigate the time evolution of the atom initially in the first excited level. In a very large cavity (free space) for a long elapsed time, the atom decays and the value of its occupation number is the physically expected one at a given temperature. For a small cavity the excited atom never completely decays and the stability rate depends on temperature.
\end{abstract}

DOI: 10.1103/PhysRevA.81.032119

PACS number(s): 03.65.Ca

\section{INTRODUCTION}

The coupling of a single atom with radiation in a cavity has been the subject of several experiments over the past decades [1-3]. The effects of confinement on atoms within metallic cavities with size of the order of microns have been investigated [1] using laser spectroscopic measurements of the interaction between a single Rydberg atom and a gold cavity. From these, one infers that a simple electrostatic model of the atom-cavity interaction is correct when the cavity is small enough. On the other hand, different processes have been developed for controlling the coupling rate, such as trapping and cooling the atom to the ground state [3-5]. Recently, with the use of fiber-based cavity and atom-chip devices [6-8], other experimental results have been found and new possibilities have been brought about, in particular to analyze temperature effects on the spontaneous emission of atoms in cavities. Thermal effects change, for instance, the lifetime of Rydberg states [9] as a direct consequence of a redistribution of the atom levels due to the thermal radiation.

Temperature effects for atom-cavity quantum electrodynamics (QED) have also been considered from a theoretical point of view for different systems. For instance, the role of the cavity temperature on an effective two-level atom undergoing two-photon transitions in a high- $Q$ cavity has been investigated [10]. The quantum statistical properties of the field and the dynamical properties of the atom are studied and compared with those for an atom making one-photon transitions between

\footnotetext{
*khanna@phys.ualberta.ca

†adolfo@cbpf.br

†jmalboui@ufba.br

§asantana@fis.unb.br
}

the two levels. A more complex system, but theoretically connected with atoms coupled to an environment at finite temperature, is an entangled pair of two-level systems [11]. In this case, each subsystem is interacting with a reservoir at finite temperature. As a result, it is observed that the temperature has a dramatic effect on the entanglement properties of the system. In a similar context, the inhibition of spontaneous emission in Fermi gases is taken as a quantum-statistical analog of inhibition of spontaneous emission by an excited atom in a cavity. It has been shown [12] that the existence of nontrivial effects at finite temperature for the inhibition of spontaneous emission in Fermi gases should imply an analogous effect for excited confined atoms.

Using the theoretical approach to describe an atom-cavity QED system is a complicated task, in particular regarding inhibition of spontaneous emission $[13,14]$; it demands high orders in perturbative calculations. This aspect makes the Feynman diagram approach practically unreliable and thus leads to considering a nonperturbative treatment of such systems using the semiqualitative idea of a dressed atom [15]. However, such an approach is also intricate due to nonlinear effects. A way to circumvent these problems is to assume that under certain conditions the coupled atom-electromagnetic field system may be approximated by a system composed of a harmonic oscillator coupled linearly to the field modes through an effective coupling constant $g$. This is the case for linear response theory in QED, where the electric dipole interaction gives the leading contribution to the radiation process [16,17]. Simplified theoretical models can be used to describe atoms in cavities, with the expectation that it will fit the experimental results [1]. This also permits a better understanding of the need for a nonperturbative analytical treatment of coupled systems, which is the basic aspect underlying the idea of dressed quantum mechanical operators. 
Regarding the nature of a perturbative theory, the analysis is carried out by considering bare, noninteracting, fields. The interaction is taken into account order by order in powers of the coupling constant. However, the idea of a bare particle associated to a bare matter field is actually an artifact. A charged physical particle is always coupled to the gauge field; that is, it is always "dressed" by a cloud of quanta of the gauge field (photons, in the case of electrodynamics). Beyond that, there are situations where the use of perturbation theory is not advisable, as in the low-energy domain of quantum chromodynamics and resonant effects in atomic physics [15]. In this latter case, the semiqualitative idea of a dressed atom has been used for a long time.

An alternative approach, using a simplified model for a radiating atom, has been introduced $[18,19]$. This method has been employed in several cases [20-23] in agreement with experiments [24] and uses dressed objects, such as dressed states and dressed or renormalized coordinates, directly. These dressed states can be seen as a rigorous version of the general idea of dressed quantum particles in the framework of the adopted model. We explore this method in the present article, and for this reason it is worth emphasizing that our dressed states do not correspond to the semiqualitative notion of dressed atoms currently used in atomic physics. The latter is associated with the eigenstates of the system, while the dressed states considered here are related to renormalized coordinates with a nonunitary time evolution.

We consider as a model, in the harmonic approximation, an atom coupled to an environment modeled by an infinite set of pointlike harmonic oscillators (the field modes). A model of this type, describing a linear coupling of a particle with an environment, has been used in several situations, as in the study of quantum Brownian motion of a particle with the path-integral formalism [25-28]. The dressed-state formalism explored here is restricted to this specific model; nevertheless, the underlying idea is quite general and can be adapted for other situations, including nonlinear coupling [22], entangled states [29], and thermal effects. This latter aspect, as a goal, is addressed in the present work by analyzing how the well-established stability of cold confined atoms is changed by heating. We investigate the effects in a range of temperatures going from room temperature up to $T=10^{5} \mathrm{~K}(\sim 8.4 \mathrm{eV})$, which is below the ionization temperature of $13.6 \mathrm{eV}$ for the hydrogen atom, and so we can speak of the atom not being dissociated. Some effects on the average occupation number at this temperature are studied with respect to the corresponding zero- and room-temperature values.

The presentation is organized in the following way. In Sec. II the model is defined, in Sec. III the renormalized coordinates and dressed states are discussed, in Sec. IV the thermal effect in a small cavity is analyzed, and in Sec. V we present our final concluding remarks.

\section{THE MODEL}

We start by considering a bare atom approximated by a harmonic oscillator described by the bare coordinate and momentum $q_{0}, p_{0}$, respectively, having bare frequency $\omega_{0}$, linearly coupled to a set of $N$ other harmonic oscillators (the environment) described by bare coordinate and momenta $q_{k}, p_{k}$ respectively, with frequencies $\omega_{k}, k=1,2, \ldots, N$. The limit $N \rightarrow \infty$ will be taken later. The whole system is supposed to reside inside a spherical cavity of radius $R$ in thermal equilibrium with the environment at a temperature $T=\beta^{-1}\left(k_{B}\right.$, the Boltzmann constant is taken equal to 1$)$.

The Hamiltonian for such a system is written in the form

$$
H=\frac{1}{2}\left[p_{0}^{2}+\omega_{0}^{2} q_{0}^{2}+\sum_{k=1}^{N}\left(p_{k}^{2}+\omega_{k}^{2} q_{k}^{2}\right)\right]-q_{0} \sum_{k=1}^{N} c_{k} q_{k},
$$

where the $c_{k}$ 's are coupling constants. In the limit $N \rightarrow \infty$, we recover the case of an atom coupled to the environment, after redefining divergent quantities, in a manner analogous to mass renormalization in field theories.

The Hamiltonian (1) is transformed to the principal axis by means of a point transformation,

$$
q_{\mu}=\sum_{r=0}^{N} t_{\mu}^{r} Q_{r}, \quad p_{\mu}=\sum_{r=0}^{N} t_{\mu}^{r} P_{r},
$$

where $\mu=(0,\{k\}) k=1,2, \ldots, N$, performed by an orthonormal matrix $T=\left(t_{\mu}^{r}\right)$. The subscripts $\mu=0$ and $\mu=k$ refer, respectively, to the atom and the harmonic modes of the reservoir and $r$ refers to the normal modes. In terms of normal momenta and coordinates, the transformed Hamiltonian reads

$$
H=\frac{1}{2} \sum_{r=0}^{N}\left(P_{r}^{2}+\Omega_{r}^{2} Q_{r}^{2}\right)
$$

where the $\Omega_{r}$ 's are the normal frequencies corresponding to the stable collective oscillation modes of the coupled system. It can be shown [18] that

$$
t_{k}^{r}=\frac{c_{k}}{\omega_{k}^{2}-\Omega_{r}^{2}} t_{0}^{r}, \quad t_{0}^{r}=\left[1+\sum_{k=1}^{N} \frac{c_{k}^{2}}{\left(\omega_{k}^{2}-\Omega_{r}^{2}\right)^{2}}\right]^{-\frac{1}{2}},
$$

with the condition

$$
\omega_{0}^{2}-\Omega_{r}^{2}=\sum_{k=1}^{N} \frac{c_{k}^{2}}{\omega_{k}^{2}-\Omega_{r}^{2}} .
$$

To correctly describe the coupling of the atom with the field, we take

$$
c_{k}=\eta \omega_{k}, \quad \eta=\sqrt{2 g \Delta \omega},
$$

where $g$ is a constant with a dimension of frequency. It measures the strength of the coupling; $\Delta \omega=\pi c / R$ is the interval between two neighboring frequencies of the reservoir and frequencies of the field modes are given by [18]

$$
\omega_{k}=k \Delta \omega=k \frac{\pi c}{R} \text {. }
$$

The sum in Eq. (4) diverges for $N \rightarrow \infty$. This makes the equation meaningless, unless a renormalization procedure, analogous to mass renormalization in field theories, is implemented [30]. Adding and subtracting a term $\eta^{2} \Omega_{r}^{2}$ in the numerators of the right-hand side in Eq. (4), we have

$$
\bar{\omega}^{2}-\Omega_{r}^{2}=\eta^{2} \Omega_{r}^{2} \sum_{k=1}^{\infty} \frac{1}{\omega_{k}^{2}-\Omega_{r}^{2}},
$$


where we define the renormalized frequency

$$
\bar{\omega}^{2}=\omega_{0}^{2}-\delta \omega^{2}=\lim _{N \rightarrow \infty}\left(\omega_{0}^{2}-N \eta^{2}\right)
$$

We find that the addition of a counterterm $-\delta \omega^{2} q_{0}^{2}$ in the Hamiltonian Eq. (1) compensates for the divergence of $\omega_{0}^{2}$ in such a way as to leave a finite, physically meaningful, renormalized frequency $\bar{\omega}$.

Using the formula

$$
\sum_{k=1}^{\infty} \frac{1}{k^{2}-u^{2}}=\frac{1}{2 u^{2}}-\frac{\pi}{2 u} \cot (\pi u)
$$

Eq. (7) can be rewritten as (dropping the label for the eigenfrequencies)

$$
\cot \left(\frac{R \Omega}{c}\right)=\frac{\Omega}{\pi g}+\frac{c}{R \Omega}\left(1-\frac{R \bar{\omega}^{2}}{\pi g c}\right) .
$$

This gives an infinity of solutions. The spectrum of the collective normal modes is denoted by $\Omega_{r} ; r=0,1,2, \ldots$. The transformation matrix elements are [18]

$$
\begin{gathered}
t_{0}^{r}=\frac{\eta \Omega_{r}}{\sqrt{\left(\Omega_{r}^{2}-\bar{\omega}^{2}\right)^{2}+\frac{\eta^{2}}{2}\left(3 \Omega_{r}^{2}-\bar{\omega}^{2}\right)+\pi^{2} g^{2} \Omega_{r}^{2}}}, \\
t_{k}^{r}=\frac{\eta \omega_{k}}{\omega_{k}^{2}-\Omega_{r}^{2}} t_{0}^{r} .
\end{gathered}
$$

Unless explicitly stated, the limit $N \rightarrow \infty$ is understood in the following.

\section{RENORMALIZED COORDINATES AND DRESSED STATES}

Let us consider the eigenstates of our system, $\left|n_{0}, n_{1}, n_{2} \ldots\right\rangle$, represented by the normalized eigenfunctions in terms of the normal coordinates $\left\{Q_{r}\right\}$,

$$
\phi_{n_{0} n_{1} n_{2} \ldots}(Q)=\prod_{s}\left[\sqrt{\frac{2^{n_{s}}}{n_{s} !}} H_{n_{s}}\left(\sqrt{\frac{\Omega_{s}}{\hbar}} Q_{s}\right)\right] \Gamma_{0},
$$

where $H_{n_{s}}$ stands for the $n_{s}$ th Hermite polynomial and $\Gamma_{0}$ is the normalized ground-state eigenfunction,

$$
\Gamma_{0}(Q)=\mathcal{N} \exp \left[-\frac{1}{2 \hbar} \sum_{r=0}^{\infty} \Omega_{r} Q_{r}^{2}\right] .
$$

We introduce dressed or renormalized coordinates $q_{0}^{\prime}$ and $\left\{q_{k}^{\prime}\right\}$ for, respectively, the dressed atom and the dressed field, defined by

$$
\sqrt{\bar{\omega}_{\mu}} q_{\mu}^{\prime}=\sum_{r} t_{\mu}^{r} \sqrt{\Omega_{r}} Q_{r}
$$

where $\bar{\omega}_{\mu}=\left\{\bar{\omega}, \omega_{k}\right\}$. In terms of renormalized coordinates, we define for the time $\tau=0$, the dressed states, $\left|\kappa_{0}, \kappa_{1}, \kappa_{2} \ldots\right\rangle$ by means of the complete orthonormal functions

$$
\psi_{\kappa_{0} \kappa_{1} \ldots}\left(q^{\prime}\right)=\prod_{\mu}\left[\sqrt{\frac{2^{\kappa_{\mu}}}{\kappa_{\mu} !}} H_{\kappa_{\mu}}\left(\sqrt{\frac{\bar{\omega}_{\mu}}{\hbar}} q_{\mu}^{\prime}\right)\right] \Gamma_{0},
$$

where $q_{\mu}^{\prime}=\left\{q_{0}^{\prime}, q_{k}^{\prime}\right\}$ and $\bar{\omega}_{\mu}=\left\{\bar{\omega}, \omega_{k}\right\}$. Notice that the ground state, $\Gamma_{0}$, in the above equation is the same as in Eq. (12). The invariance of the ground state is due to our definition of renormalized coordinates given by Eq. (14). In fact, we get the normal coordinates $Q_{r}$ in terms of the renormalized ones from Eq. (14). Replacing them in Eq. (13) we find that the ground state in terms of the renormalized coordinates has the form

$$
\Gamma_{0}\left(q^{\prime}\right)=\mathcal{N} \exp \left[-\frac{1}{2 \hbar} \sum_{\mu=0}^{\infty} \bar{\omega}_{\mu} q_{\mu}^{\prime 2}\right] .
$$

Each function $\psi_{\kappa_{0} \kappa_{1} \ldots}\left(q^{\prime}\right)$ describes a state in which the dressed oscillator $q_{\mu}^{\prime}$ is in its $\kappa_{\mu}$ th excited state.

It is worth noting that our renormalized coordinates are objects different from both the bare coordinates $q$ and the normal coordinates $Q$. Our dressed states, although being collective objects, should not be confused with the eigenstates given by Eq. (12). While the eigenstates $\phi$ are stable, all the dressed states $\psi$ are unstable, except for the ground state $\Gamma_{0}$. The need for renormalized coordinates can be understood by considering what happens if we write Eq. (15) in terms of the bare coordinates $q$. In the absence of interaction, the bare states are stable since they are eigenfuntions of the free Hamiltonian. However, with the onset of the interaction they all become unstable. We know that the excited states are unstable, and we also know from experiment that the atom in its ground state is stable, thus in contradiction with the description of our model in terms of the bare coordinates. The solution we find to this paradox is just the introduction of the renormalized coordinates $q^{\prime}$ as the physically meaningful ones. This can be seen as an analog of the wave-function renormalization in quantum field theory, which justifies the denomination of renormalized to the new coordinates $q^{\prime}$.

We intend to divide the system into the dressed atom and the dressed environment by means of the renormalized coordinates $q_{0}^{\prime}$ and $q_{j}^{\prime}$ associated, respectively, to the dressed atom and to the dressed oscillators composing the environment. These coordinates allow a natural division of the system into the dressed (physically observed) atom and into the dressed environment. The dressed atom will contain automatically all the effects of the environment on it. In order to see this more precisely, let us write the renormalized coordinates in terms of the bare coordinates,

$$
q_{\mu}^{\prime}=\sum_{\nu} \alpha_{\mu \nu} q_{\nu} ; \quad \alpha_{\mu \nu}=\frac{1}{\sqrt{\bar{\omega}_{\mu}}} \sum_{r} t_{\mu}^{r} t_{\nu}^{r} \sqrt{\Omega_{r}},
$$

which in the limit $R \rightarrow \infty$ become

$$
q_{0}^{\prime}=\frac{1}{\bar{\omega}} \int_{0}^{\infty} \frac{2 g \Omega^{2} \sqrt{\Omega} d \Omega}{\left(\Omega^{2}-\bar{\omega}^{2}\right)^{2}+\pi^{2} g^{2} \Omega^{2}} ; \quad q_{k}^{\prime}=q_{k} .
$$

It is interesting to compare Eqs. (17) and (18). In the case of Eq. (17) for finite $R$, the coordinates $q_{0}^{\prime}$ and $q_{k}^{\prime}$ are all dressed in the sense that they are all collective; both the field modes and the atom cannot be separated in this language. In the limit $R \rightarrow \infty$, we see from Eq. (18) that the coordinate $q_{0}^{\prime}$ describes the atom modified by the presence of the field in an indisoluble way; the atom is always dressed by the field. On the other hand, the dressed harmonic modes of the field, described by the coordinates $q_{k}^{\prime}$, coincide with the field modes; in other words, the field retains in this limit its proper identity, while the atom is always accompanied by a cloud of field quanta. Therefore, we identify the coordinate $q_{0}^{\prime}$ as the coordinate describing the atom dressed by its proper field, the whole system being 
divided into dressed atom and field, without recourse to the concept of interaction between them, this being absorbed in the dressing cloud of the atom. If the cavity is finite, but much larger than the atom (for instance $R \sim 10^{-6} \mathrm{~m}$ ), we can keep the same language. We can speak of the dressed atom and the field, since the infinity of the field modes do not interact among themselves and are weakly dressed by the single atom.

As mentioned previously, the dressed states given by Eq. (15) evolve in time in a well-defined way; they are linear combinations of the (stable) eigenstates (12) defined in terms of the normal modes. Explicit formulas for coefficients of these combinations are obtained for the interesting physical situations [18]. This gives a complete and rigorous definition of our dressed states. Moreover, our dressed states have the interesting property of distributing the energy initially in a particular dressed state, among itself and all other dressed states with well-defined probability amplitudes [18]. We choose these dressed states as physically meaningful and we test successfully this hypothesis by studying the radiation process by an atom in a cavity. In both cases, a very large and a very small cavity, our results are in agreement with experimental observations $[18,19,21]$.

In this framework, we write the physical states in terms of dressed annihilation and creation operators $a_{\mu}^{\prime}$ and $a_{\mu}^{\prime \dagger}$ defined in terms of renormalized coordinates and momenta in the usual way,

$$
\begin{aligned}
& a_{\mu}^{\prime}=\sqrt{\frac{\bar{\omega}_{\mu}}{2}} q_{\mu}^{\prime}+\frac{i}{\sqrt{2 \bar{\omega}_{\mu}}} p_{\mu}^{\prime}, \\
& a_{\mu}^{\prime \dagger}=\sqrt{\frac{\bar{\omega}_{\mu}}{2}} q_{\mu}^{\prime}-\frac{i}{\sqrt{2 \bar{\omega}_{\mu}}} p_{\mu}^{\prime} .
\end{aligned}
$$

Then the initial dressed density operator corresponding to the thermal bath is given by

$$
\rho_{\beta}^{\prime}=\frac{1}{Z_{\beta}^{\prime}} \exp \left[-\hbar \beta \sum_{k=1}^{\infty} \omega_{k}\left(a_{k}^{\prime \dagger} a_{k}^{\prime}+\frac{1}{2}\right)\right],
$$

with $Z_{\beta}^{\prime}=\prod_{k} z_{\beta}^{k \prime}$ being the partition function of the dressed reservoir, where

$$
z_{\beta}^{k \prime}=\operatorname{Tr}\left[e^{-\hbar \beta \omega_{k}\left(a_{k}^{\prime \dagger} a_{k}^{\prime}+1 / 2\right)}\right] .
$$

The system evolves with time $(\tau)$. The time-dependent $d r e s s e d$ occupation numbers are defined as

$$
n_{\mu}^{\prime}(\tau)=\operatorname{Tr}\left[a_{\mu}^{\prime \dagger}(\tau) a_{\mu}^{\prime}(\tau) \rho_{0}^{\prime} \otimes \rho_{\beta}^{\prime}\right]
$$

(the prime is to clearly distinguish the dressed quantities from the bare ones), where $\rho_{0}^{\prime}$ is the density operator for the dressed atom and $a_{\mu}^{\prime}(\tau)$ and $a_{\mu}^{\prime \dagger}(\tau)$ are the time-dependent creation and annihilation operators.

The time evolution of the dressed annihilation operator is given by

$$
\frac{d}{d \tau} a_{\mu}^{\prime}(\tau)=i\left[H, a_{\mu}^{\prime}(\tau)\right]
$$

and a similar equation for $a_{\mu}^{\prime \dagger}(\tau)$. We solve this equation with the initial condition at time $\tau=0$,

$$
a_{\mu}^{\prime}(0)=\sqrt{\frac{\omega_{\mu}}{2}} q_{\mu}^{\prime}+\frac{i}{\sqrt{2 \omega_{\mu}}} p_{\mu}^{\prime},
$$

which, in terms of bare coordinates, becomes

$$
a_{\mu}^{\prime}(0)=\sum_{r, \nu=0}^{N}\left(\sqrt{\frac{\Omega_{r}}{2}} t_{\mu}^{r} t_{\nu}^{r} q_{\nu}+\frac{i t_{\mu}^{r} t_{\nu}^{r}}{\sqrt{2 \Omega_{r}}} p_{\nu}\right) .
$$

We assume a solution for $a_{\mu}^{\prime}(\tau)$ of the type

$$
a_{\mu}^{\prime}(\tau)=\sum_{\nu=0}^{\infty}\left[\dot{B}_{\mu \nu}^{\prime}(\tau) q_{\nu}+B_{\mu \nu}^{\prime}(\tau) p_{\nu}\right] .
$$

Using Eq. (1) we find

$$
B_{\mu \nu}^{\prime}(\tau)=\sum_{r=0}^{\infty} t_{\nu}^{r}\left(a_{\mu}^{\prime r} e^{i \Omega_{r} \tau}+b_{\mu}^{\prime r} e^{-i \Omega_{r} \tau}\right) .
$$

The initial conditions for $B_{\mu \nu}^{\prime}(\tau)$ and $\dot{B}_{\mu \nu}^{\prime}(\tau)$ are obtained by setting $\tau=0$ in Eq. (26) and comparing with Eq. (25); then

$$
\begin{gathered}
B_{\mu \nu}^{\prime}(0)=i \sum_{r=0}^{\infty} \frac{t_{\mu}^{r} t_{v}^{r}}{\sqrt{2 \Omega_{r}}}, \\
\dot{B}_{\mu \nu}^{\prime}(0)=\sum_{r=0}^{\infty} \sqrt{\frac{\Omega_{r}}{2}} t_{\mu}^{r} t_{\nu}^{r} .
\end{gathered}
$$

Using these initial conditions and the orthonormality of the matrix $\left\{t_{\mu}^{r}\right\}$, we obtain $a_{\mu}^{\prime r}=0, b_{\mu}^{\prime r}=i t_{\mu}^{r} / \sqrt{2 \Omega_{r}}$. Replacing these values for $a_{\mu}^{\prime r}$ and $b_{\mu}^{\prime r}$ in Eq. (27), we get

$$
B_{\mu \nu}^{\prime}(\tau)=i \sum_{r=0}^{\infty} \frac{t_{\mu}^{r} t_{v}^{r}}{\sqrt{2 \Omega_{r}}} e^{-i \Omega_{r} \tau}
$$

We have

$$
\begin{aligned}
a_{\mu}^{\prime}(\tau) & =\sum_{r, v=0}^{\infty} t_{\mu}^{r} t_{\nu}^{r}\left(\sqrt{\frac{\Omega_{r}}{2}} q_{\nu}+\frac{i}{\sqrt{2 \Omega_{r}}} p_{\nu}\right) e^{-i \Omega_{r} \tau}, \\
& =\sum_{r, v=0}^{N} t_{\mu}^{r} t_{\nu}^{r}\left(\sqrt{\frac{\omega_{v}}{2}} q_{\nu}^{\prime}+\frac{i}{\sqrt{2 \omega_{\nu}}} p_{\nu}^{\prime}\right) e^{-i \Omega_{r} \tau}, \\
& =\sum_{\nu=0}^{\infty} f_{\mu \nu}(\tau) a_{\nu}^{\prime},
\end{aligned}
$$

where

$$
f_{\mu \nu}(\tau)=\sum_{r=0}^{\infty} t_{\mu}^{r} t_{\nu}^{r} e^{-i \Omega_{r} \tau},
$$

with $\mu, v=0,\{k\}, k=1,2, \ldots$.

This leads to the time evolution equation for the dressed occupation number of the atom $[31,32]$,

$$
n_{0}^{\prime}(\tau)=\left|f_{00}(\tau)\right|^{2} n_{0}^{\prime}+\sum_{k=1}^{\infty}\left|f_{0 k}(\tau)\right|^{2} n_{k}^{\prime},
$$

where $n_{0}^{\prime}$ stands for the occupation number at $\tau=0$.

\section{THERMAL EFFECTS IN A SMALL CAVITY}

Now we consider the weak coupling régime, defined by

$$
g=\bar{\omega} \alpha,
$$

where $\alpha$ is the fine-structure constant. 
With $\eta=\sqrt{2 g \pi c / R}$ and defining the dimensionless parameter

$$
\delta=\frac{g}{\Delta \omega}=\frac{g R}{\pi c}
$$

Eq. (10) becomes

$$
\cot \left(\frac{\pi \Omega \delta}{g}\right)=\frac{\Omega}{\pi g}+\frac{g}{\pi \delta \Omega}\left(1-\frac{\delta \bar{\omega}^{2}}{g^{2}}\right) .
$$

Let us consider the right-hand side of Eq. (36) such that

$$
\frac{\delta \bar{\omega}^{2}}{g^{2}}>1
$$

In the weak coupling regime, this corresponds to a value of $\delta \gtrsim 5.3 \times 10^{-5}$. For a frequency $\bar{\omega}=4.0 \times 10^{14} / \mathrm{s}$ (in the visible red) this gives a condition on the cavity size of $R \gtrsim 1.7 \times 10^{-8} \mathrm{~m}$. Then the general behavior of solutions of Eq. (36) is illustrated in Fig. 1. We find that all but one of the eigenfrequencies are very close to the frequencies of the field modes, $\omega_{k}$, given by Eq. (6). Then we label solutions for the eigenfrequencies $\Omega_{r}$ as $\Omega_{0}, \Omega_{k}$, and $k=1,2, \ldots$ The solutions $\Omega_{k}$ of Eq. (36) are

$$
\Omega_{k}=\frac{g}{\delta}\left(k+\epsilon_{k}\right), \quad k=1,2, \ldots,
$$

with $0<\epsilon_{k}<1$, satisfying the equation

$$
\cot \left(\pi \epsilon_{k}\right)=\frac{1}{\pi \delta}\left(k+\epsilon_{k}\right)+\frac{1}{\pi\left(k+\epsilon_{k}\right)}\left(1-\frac{\delta \bar{\omega}^{2}}{g^{2}}\right) .
$$

Since every $\epsilon_{k}$ is much smaller than 1, Eq. (39) can be linearized in $\epsilon_{k}$, giving

$$
\epsilon_{k}=\frac{\delta g^{2} k}{g^{2} k^{2}-\delta^{2} \bar{\omega}^{2}} .
$$

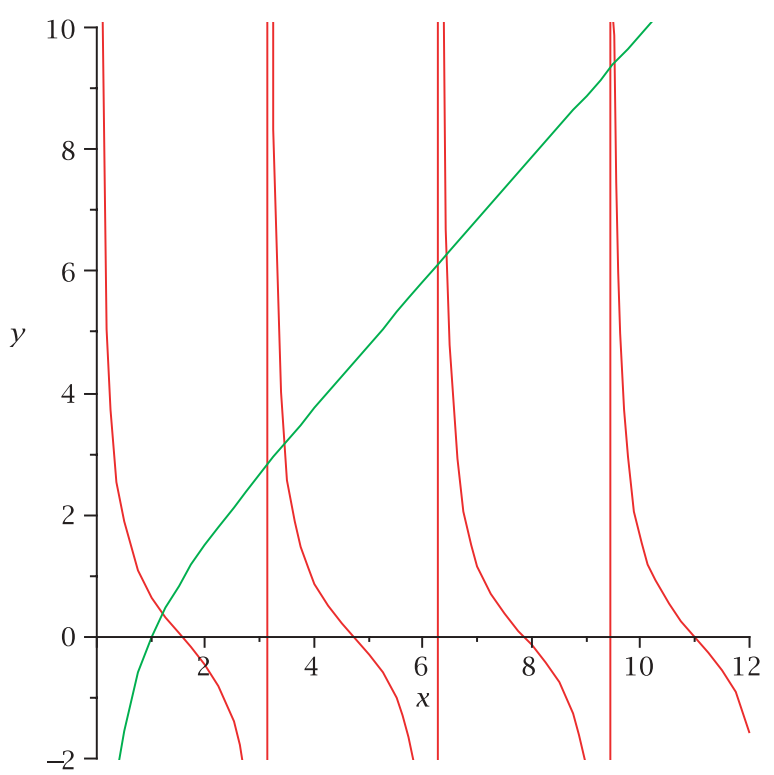

FIG. 1. (Color online) Solutions of Eq. (36), with $y=\cot (x)$ and $x=\pi \Omega \delta / g$, for cavities satisfying the condition given in Eq. (37). The asymptotes of the cotangent curve correspond to the frequencies of the field modes $\omega_{k}$.
The eigenfrequencies, $\Omega_{k}$, are obtained by solving Eqs. (38) and (39) or (40).

The lowest eigenfrequency, $\Omega_{0}$, is obtained by assuming that it satisfies the condition $\Omega_{0} R / c=\Omega_{0} \pi \delta / g \ll 1$. Inserting this condition in Eq. (36) and keeping up to quadratic terms in $\Omega$, we obtain the solution for the lowest eigenfrequency,

$$
\Omega_{0} \approx \bar{\omega}\left(1-\frac{\pi^{2} \delta}{6}\right) .
$$

Consistency between Eq. (36) and the condition $\Omega_{0} R / c \ll$ 1 gives a condition on $R$, that is, $R \ll(c / g) \lambda$, with $\lambda=(\pi / 2)(g / \bar{\omega})^{2}$.

Let us first determine the temperature-independent term $\left|f_{00}(\tau)\right|^{2} n_{0}^{\prime}$ in Eq. (33), considering that the dressed atom is initially (at $\tau=0$ ) in the first excited level, that is $n_{0}^{\prime}=1$. We evaluate $\left(t_{0}^{0}\right)^{2}$ and $\left(t_{0}^{k}\right)^{2}$ from Eqs. (12), (38), (40), and (41) to find

$$
\left(t_{0}^{k}\right)^{2} \approx \frac{2 g R}{\pi c k^{2}}=\frac{2 \delta}{k^{2}},
$$

and then using the normalization condition $\sum_{r=0}^{\infty}\left(t_{0}^{r}\right)^{2}=1$ and $\zeta(2)=\sum_{k=1}^{\infty} k^{-2}=\pi^{2} / 6$, we have

$$
\left(t_{0}^{0}\right)^{2} \approx 1-\frac{\pi g R}{3 c}=1-\frac{\pi^{2} \delta}{3} .
$$

From Eq. (32), using the de Moivre formula, $e^{i \theta}=\cos \theta+$ $i \sin \theta$, we have

$$
\left|f_{\mu \nu}(\tau)\right|^{2}=\sum_{r, s=0}^{\infty} t_{\mu}^{r} t_{\nu}^{r} t_{\mu}^{s} t_{\nu}^{s} \cos \left(\Omega_{r}-\Omega_{s}\right) \tau .
$$

Let us assume that the thermal bath is at zero temperature, that is, all the modes of the reservoir are in the ground state $n_{k}^{\prime}=0$ for all values of $k$. Taking the preceding approximations for $t_{0}^{k}$ and $t_{0}^{0}$, we get from Eq. (33) the zero-temperature time evolution of the occupation number of the dressed atom initially in the first excited level,

$$
\begin{aligned}
\left|f_{00}(\tau)\right|^{2} \approx & \left(1-\frac{\pi^{2} \delta}{3}\right)^{2}+4 \delta\left(1-\frac{\pi^{2} \delta}{3}\right) \\
& \times \sum_{k=1}^{\infty} \frac{1}{k^{2}} \cos \left(\Omega_{k}-\Omega_{0}\right) \tau \\
& +4 \delta^{2} \sum_{k, l=1}^{\infty} \frac{1}{k^{2} l^{2}} \cos \left(\Omega_{k}-\Omega_{l}\right) \tau .
\end{aligned}
$$

This is an oscillating function which has a minimum value $\min \left(\left|f^{00}(t)\right|^{2}\right)$. Taking both cosine functions in Eq. (45) equal to -1 , we get a lower bound for $\min \left(\left|f^{00}(t)\right|^{2}\right)$ given, up to first order in $\delta$, by

$$
F(\delta)=1-\left(\frac{2 \pi^{2}}{3}-2\right) \delta .
$$

As an example we consider that the atom in the first excited state has an emission frequency $\bar{\omega} \approx 4 \times 10^{14} / \mathrm{s}$, in the visible red, and we take the radius of the confining cavity $R \approx 10^{-6} \mathrm{~m}$. With these data we get $F(\delta) \approx 0.99$, that is, a probability of $99 \%$ at zero temperature, that it will almost never decay. This shows the high stability of the system, which is confirmed by experiment $[33,34]$. 
In order to take into account the temperature effects, we must consider the second term in Eq. (33); that is, we must evaluate the quantity

$$
\left|f_{0 k}(\tau)\right|^{2}=\left|t_{0}^{0} t_{k}^{0} e^{-i \Omega_{0} \tau}+\sum_{l=1}^{\infty} t_{0}^{l} t_{k}^{l} e^{-i \Omega_{l} \tau}\right|^{2} .
$$

This is carried out by using the matrix elements obtained from Eq. (12) and the formulas for eigenfrequencies in a small cavity.

Then it is necessary to make an assumption on how the occupation numbers of the field modes depend on temperature. We assume that the thermal distribution of the occupation numbers of the field modes in the cavity follow the BoseEinstein distribution,

$$
n_{k}^{\prime}(\beta)=\frac{1}{e^{\hbar \beta \omega_{k}}-1} .
$$

This can be justified in the following way: In the case of an arbitrarily large cavity, the dressed field modes coincide with the bare ones, as can be seen from Eq. (18), and in the limit of vanishing coupling, these modes follow the BoseEinstein distribution exactly. Strictly speaking, this is not the case for the coupled system in a finite cavity. Nevertheless, in many situations this approximation is acceptable in the weak coupling regime. For instance, a cavity of radius $R \approx 10^{-6} \mathrm{~m}$ is $\sim 10^{4}$ times larger than the size of a hydrogen atom (the Bohr radius). In such a case the atom "sees" the cavity to be a very large one and, in the weak coupling regime, the approximation that the field modes follow the Bose-Einstein distribution is justified. Moreover, this leads to the correct zero-temperature limit

$$
\lim _{\beta \rightarrow \infty} n_{k}^{\prime}(\beta)=\lim _{\beta \rightarrow \infty} \frac{1}{e^{\hbar \beta \omega_{k}}-1}=0, \quad \forall k .
$$

Then from Eqs. (47) and (33), we get the time evolution of the temperature-dependent occupation number for the atom,

$$
\begin{aligned}
n_{0}^{\prime}(\tau, \beta)= & \left|f_{00}(\tau)\right|^{2} n_{0}^{\prime}+\sum_{k=1}^{\infty} \frac{1}{e^{(\hbar \beta \pi c / R) k}-1} \\
& \times\left[\left(t_{0}^{0}\right)^{2}\left(t_{k}^{0}\right)^{2}+2 \sum_{l=1}^{\infty} t_{0}^{0} t_{0}^{l} t_{k}^{0} t_{k}^{l} \cos \left(\Omega_{0}-\Omega_{l}\right) \tau\right. \\
& \left.+\sum_{l, n=1}^{\infty} t_{0}^{l} t_{0}^{n} t_{k}^{l} t_{k}^{n} \cos \left(\Omega_{l}-\Omega_{n}\right) \tau\right]
\end{aligned}
$$

where $\left|f_{00}(\tau)\right|^{2}$ is given by Eq. (45). The matrix elements $t_{k}^{0}$ and $t_{k}^{l}$ in the preceding formulas are evaluated from Eqs. (12), (38), and (41) to be

$$
t_{k}^{0}=\frac{k g^{2} \sqrt{2 \delta}}{k^{2} g^{2}-\Omega_{0}^{2} \delta^{2}} ; \quad t_{k}^{l}=\frac{2 k \delta}{k^{2}-\left(l+\epsilon_{l}\right)^{2}} \frac{1}{l} .
$$

The occupation number $n_{0}^{\prime}(\tau, \beta)$ is an oscillating function which has a minimum value, $\min \left[n_{0}^{\prime}(\tau, \beta)\right]$, that depends on the temperature $\beta^{-1}$. We can obtain a lower bound, $n_{0}^{\prime}(\beta)$, for this minimum, such that $\min \left[n_{0}^{\prime}(\tau, \beta)\right]>n_{0}^{\prime}(\beta)$, by taking

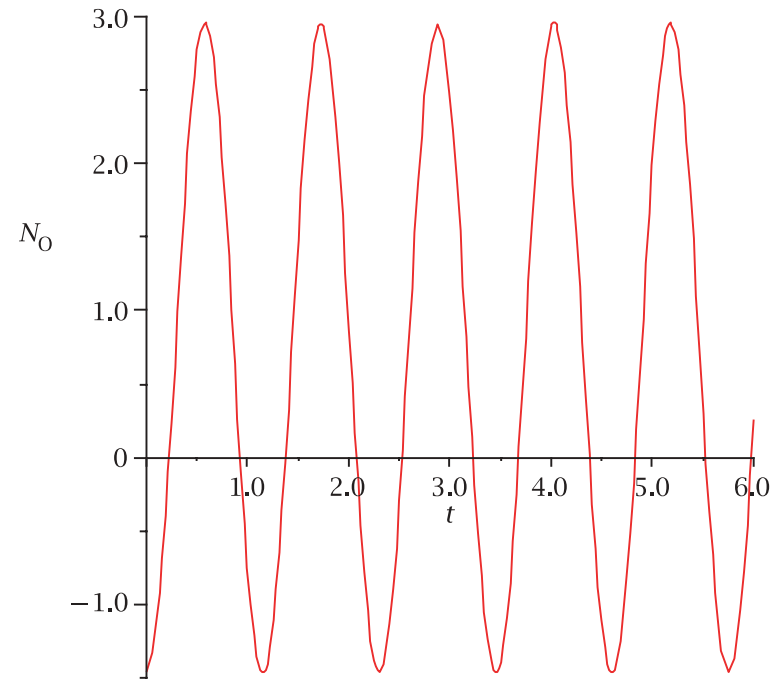

FIG. 2. (Color online) Time evolution of the thermal-dependent occupation number $n_{0}^{\prime}(\tau, \beta)$ for a temperature $T=300 \mathrm{~K}$. The scale for the vertical axis is $N_{0}=\left(n_{0}^{\prime}-1\right) \times 10^{12}$; time is in seconds $\left(\tau=t \times 10^{-14}\right)$.

both cosine functions in Eq. (49) equal to -1 :

$$
\begin{aligned}
n_{0}^{\prime}(\beta)= & F(\delta) n_{0}^{\prime}+\sum_{k=1}^{\infty} \frac{1}{e^{(\hbar \beta \pi c / R) k}-1} \\
& \times\left[\left(t_{0}^{0}\right)^{2}\left(t_{k}^{0}\right)^{2}-2 \sum_{l=1}^{\infty} t_{0}^{0} t_{0}^{l} t_{k}^{0} t_{k}^{l}-\sum_{l, n=1}^{\infty} t_{0}^{l} t_{0}^{n} t_{k}^{l} t_{k}^{n}\right] .
\end{aligned}
$$

Numerical calculation of Eqs. (49) and (51) describes how the time evolution of the occupation number and the stability of the excited atom are affected by heating. We take for the plots $n_{0}^{\prime}=1$, that is, the atom initially in the first excited level.

In Figs. 2 and 3, the time evolution of the temperaturedependent occupation number $n_{0}^{\prime}(\tau, \beta)$ is plotted for some

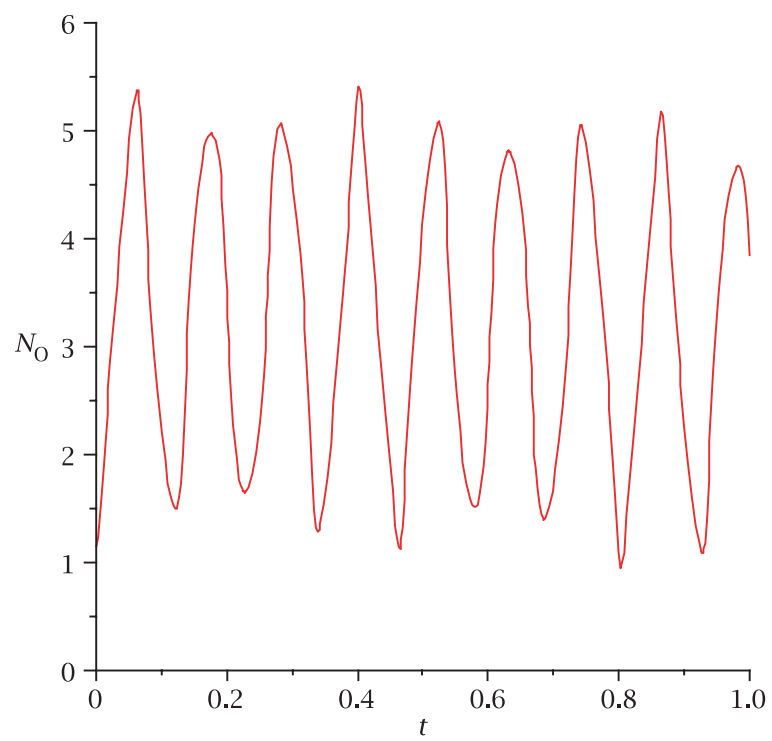

FIG. 3. (Color online) Time evolution of the thermal-dependent occupation number $n_{0}^{\prime}(\tau, \beta)$ for a temperature $T=10^{5} \mathrm{~K}$ Time is in seconds $\left(\tau=t \times 10^{-13}\right)$. 


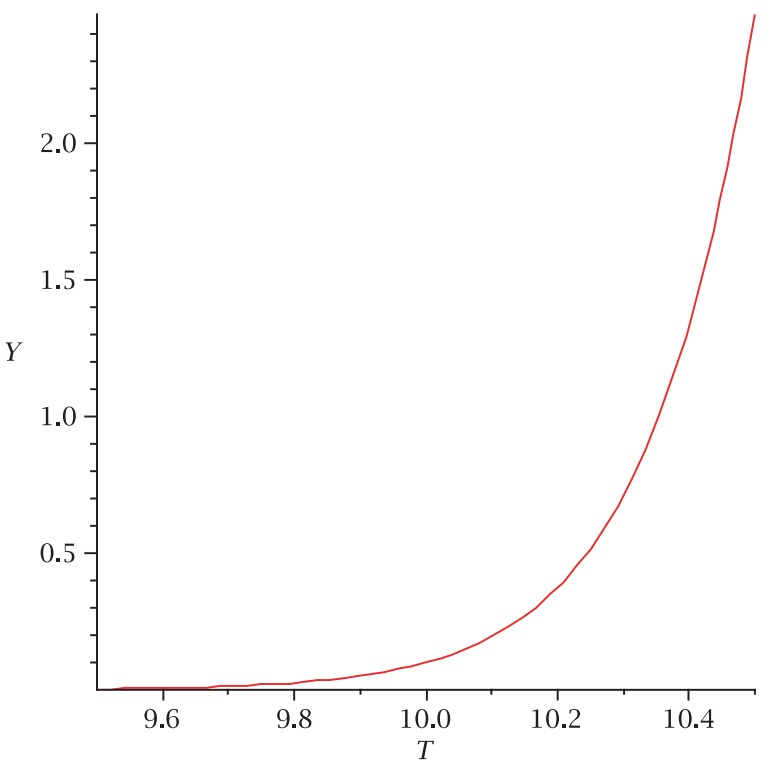

FIG. 4. (Color online) Temperature behavior of the lower bound for the minimum of $n_{0}^{\prime}(\tau, \beta), Y=n_{0}^{\prime}(\beta) \times 10^{31}$, given by Eq. (51). Temperature is given in $10 \mathrm{~K}$.

representative values of the emission frequency and temperature. In Fig. 4 the lower bound for its minimum, $n_{0}^{\prime}(\beta)$, is plotted as a function of temperature. We find from these figures that raising the temperature increases the amplitude of oscillation of the occupation number and that its minimum lower bound, $n_{0}^{\prime}(\beta)$, also grows with temperature. For a given emission frequency $\bar{\omega}=4.0 \times 10^{14} / \mathrm{s}$, the increase of the amplitude of oscillation of $n_{0}^{\prime}(\tau, \beta)$ and of its lower bound $n_{0}^{\prime}(\beta)$ are negligible for room temperatures; they are significant for high laboratory temperatures. In Fig. $3 n_{0}^{\prime}(\tau, \beta)$ is plotted for $T=$ $10^{5} \mathrm{~K}(\sim 8.4 \mathrm{eV})$; although this temperature is very high, it can, in principle, be attained in the laboratory for excited atoms. In fact, it is lower than the ionization temperature of $13.6 \mathrm{eV}$ for the hydrogen atom and still much lower than the nuclear fusion temperature of $\approx 10^{8} \mathrm{~K}(\sim 8.4 \mathrm{KeV})$. We find that the average occupation number at temperature $T=10^{5} \mathrm{~K}$ is about
3.5 times higher than the zero temperature value $n_{0}^{\prime}(\tau, T=$ $0) \approx 1$. At room temperature the occupation number will remain very close to the zero-temperature value, as shown in Fig. 2. Therefore, we find that as the temperature is raised, both the amplitude of oscillation of the occupation number and its minimum grow with respect to the zero-temperature values.

\section{CONCLUDING REMARKS}

At zero temperature, the dressed atom, initially in the first or higher excited state, can only decay, since all field modes are in the ground state. It is inhibited from decaying by confinement in a cavity of small size. However, at finite temperature, the field modes in the cavity can be in excited states with a finite probability given by the Bose-Einstein distribution function. As a consequence the dressed atom can exchange quanta with the field. This means that the thermal occupation number of excited states of the atom increases with temperature. In other words, as an effect of heating the atom will be in a higher excited state which is able to decay. However, the decay is inhibited by the confining geometry. The results presented previously give sufficient proof of these ideas.

This behavior is also to be contrasted with the situation of an arbitrarily large cavity (free space) described in [31,32]. In that case, for long times the dressed occupation number of the atom approaches smoothly an asymptotic value which is nearly the one obtained from the Bose distribution at the equilibrium temperature of the reservoir. Taking the same value as before for $\bar{\omega}$, this value is $n_{0}^{\prime}(\tau \rightarrow \infty, \beta ; \bar{\omega}, R \rightarrow \infty) \approx 0$. In that case the growth of the Bose-Einstein weight factor due to rising temperature is compensated by the lowering due to larger values of $R$, leading to an equilibrium occupation number.

\section{ACKNOWLEDGMENTS}

A.P.C.M. is grateful to the Theoretical Physics Institute, University of Alberta, for kind hospitality. The research of F.C.K. is funded by NSERC (Canada); A.P.C.M., J.M.C.M., and A.E.S. are supported by CNPq and CAPES (Brazil).
[1] E. A. Hinds, K. S. Lail, and M. Schnell, Philos. Trans. R. Soc. London A 355, 2353 (1997).

[2] H. J. Kimble, Phys. Scr. T 76, 127 (1998).

[3] J. Ye, D. W. Vernooy, and H. J. Kimble, Phys. Rev. Lett. 83, 4987 (1999).

[4] P. W. H. Pinkse, T. Fisher, P. Maunz, and G. Rempe, Nature (London) 404, 365 (2000).

[5] A. D. Boozer, A. Boca, R. Miller, T. E. Northup, and H. J. Kimble, Phys. Rev. Lett. 97, 083602 (2006).

[6] T. Steinmetz et al., Appl. Phys. Lett. 89, 111110 (2006).

[7] J. Fortágh and C. Zimmermann, Rev. Mod. Phys. 79, 235 (2007).

[8] Y. Colombe, T. Steinmetz, G. Dubois, F. Linke, D. Hunger, and J. Reichel, Nature (London) 450, 272 (2007).

[9] I. M. Beterov and I. I. Ryabtsev, JETP Lett. 69, 448 (1999).

[10] R. R. Puri and A. Joshi, Opt. Commun. 69, 367 (1989).

[11] A. Al-Qasimi and D. F. V. James, Phys. Rev. A 77, 012117 (2008).
[12] Th. Busch, J. R. Anglin, J. I. Cirac, and P. Zoller, Europhys. Lett. 44, 1 (1998).

[13] F. S. S. Rosa, T. N. C. Mendes, A. Tenorio, and C. Farina, Phys. Rev. A 78, 012105 (2008).

[14] C. Farina and T. N. C. Mendes, J. Phys. A 40, 7343 (2007).

[15] C. Cohen-Tannoudji, Atoms in Electromagnetic Fields (World Scientific, Singapore, 1994).

[16] J. M. Wylie and J. E. Sipe, Phys. Rev. A 30, 1185 (1984).

[17] W. Jhe and K. Jang, Phys. Rev. A 53, 1126 (1996).

[18] N. P. Andion, A. P. C. Malbouisson, and A. Mattos Neto, J. Phys. A 34, 3735 (2001); A. P. C. Malbouisson, Report Instituto Balseiro/CAB Bariloche, CAB/1971/13 September 1971 (unpublished).

[19] A. P. C. Malbouisson, Ann. Phys. (NY) 308, 373 (2003).

[20] G. Flores-Hidalgo, A. P. C. Malbouisson, and Y. W. Milla, Phys. Rev. A 65, 063414 (2002). 
[21] G. Flores-Hidalgo and A. P. C. Malbouisson, Phys. Rev. A 66, 042118 (2002).

[22] G. Flores-Hidalgo and A. P. C. Malbouisson, Phys. Lett. A 337, 37 (2005).

[23] G. Flores-Hidalgo, C. A. Linhares, A. P. C. Malbouisson, and J. M. C. Malbouisson, J. Phys. A 41, 075404 (2008).

[24] Note that the experimental size for a cavity, of the order of microns [1], is precisely of the order of magnitude of the cavity that some of us have used to investigate the stability of excited atoms at zero temperature in [20,21], with a good agreement with experiment. This is also the size of the cavity that we estimate for the validity of the approximation of the Bose-Einstein distribution in the present article.

[25] W. G. Unruh and W. H. Zurek, Phys. Rev. D 40, 1071 (1989).

[26] B. L. Hu, J. P. Paz, and Y. Zhang, Phys. Rev. D 45, 2843 (1992).
[27] A. O. Caldeira and A. J. Leggett, Ann. Phys. (NY) 149, 374 (1983).

[28] M. Rosenau da Costa, A. O. Caldeira, S. M. Dutra, and H. Westfahl Jr., Phys. Rev. A 61, 022107 (2000).

[29] E. R. Granhen, C. A. Linhares, A. P. C. Malbouisson, and J. M. C. Malbouisson, e-print arXiv:0910.2679.

[30] W. Thirring and F. Schwabl, Ergeb. Exakt. Naturw. 36, 219 (1964).

[31] G. Flores-Hidalgo, A. P. C. Malbouisson, J. M. C. Malbouisson, Y. W. Milla, and A. E. Santana, Phys. Rev. A 79, 032105 (2009).

[32] F. C. Khanna, A. P. C. Malbouisson, J. M. C. Malbouisson, and A. E. Santana, Thermal Quantum Field Theory: Algebraic Aspects and Applications (World Scientific, Singapore, 2009).

[33] W. Jhe, A. Anderson, E. A. Hinds, D. Meschede, L. Moi, and S. Haroche, Phys. Rev. Lett. 58, 666 (1987).

[34] R. G. Hulet, E. S. Hilfer, and D. Kleppner, Phys. Rev. Lett. 55, 2137 (1985). 